\title{
Red de maestros en educación ambiental: un espacio de formación de formadores en Bogotá, Colombia
}

TEACHERS' GROUP IN ENVIRONMENTAL EDUCATION: A SPACE OF FORMATION OF

FORMING IN BOGOTA, COLOMBIA

REDE DE MAESTROS EM EDUCAÇÃO AMBIENTAL: UM ESPAÇO DE FORMAÇÃO DE

FORMADORES EM BOGOTA, COLOMBIA

\section{Carmen Zamudio Rodríguez* / crzamudior@udistrital.edu.co}

\section{Resumen}

Este trabajo documenta algunas experiencias pedagógicas que se han implementado en el marco de la Red de Maestros en Educación Ambiental del Distrito Capital, con el fin de contribuir a la formación conceptual de los docentes de colegios oficiales que participan en el proceso de conformación de esta red. Por lo tanto, empleando elementos de investigación cualitativa, se indagan concepciones de los maestros sobre ambiente, educación ambiental y problemática ambiental, para, a partir de estas representaciones, establecer una línea de base y diseñar e implementar una estrategia metodológica de formación docente.

\section{Summary}

This paper documents some educational experiences that have been implemented within the framework of the Teacher's Environmental Education Network of the Capital District, in order to contribute to the conceptual training of teachers in official schools participating in the process of formation of this network. For that reason, using elements of qualitative research, here conceptions of teachers on environment, environmental problematic and environmental education are investigated; from these representations, a baseline is established and is designs and implements a methodological strategy of teacher training.

\section{Resumo}

Este trabalho documenta algumas experiências pedagógicas que se implementaram no marco da Rede de Maestros em Educação Ambiental do Distrito Capital, com o fim de contribuir à formação conceptual dos docentes de colégios oficiais que participam no processo de conformación desta rede. Portanto, empregando elementos de investigação cualitativa, se indagan concepções dos maestros sobre ambiente, educação ambiental e problemática ambiental, para, a partir destas representações, estabelecer uma linha de base e desenhar e implementar uma estratégia metodológica de formação docente.

\section{Palabras clave}

Formación docente, educación ambiental, redes de maestros, red en educación ambiental, representaciones sociales de ambiente y educación ambiental

\section{Keywords}

Teacher training, environmental education, networking of teachers, environmental education network

Palavras Chave

Formação docente, educação ambiental, redes de maestros, rede em educação ambiental, representações sociais de ambiente e educação ambiental

\footnotetext{
** Profesora de la Especialización en Educación y Gestión Ambiental, Facultad de Ciencias y Educación de la Universidad Distrital Francisco José de Caldas. Magíster en Medio Ambiente y Desarrollo del Instituto de Estudios Ambientales (IDEA) de la Universidad Nacional de Colombia.
}

Fecha de recepción: 28 de noviembre de 2011 / Fecha de aprobación: 8 de junio de 2012 


\section{Introducción}

La Red de Maestros en Educación Ambiental del Distrito Capital, es un proyecto que se viene llevando a cabo en la ciudad de Bogotá desde finales del año 2009, a propósito de la implementación del Plan Sectorial 2008-2012 de la Secretaría de Educación Distrital (SED), denominado "Educación de Calidad para una Bogotá Positiva". Esta propuesta corresponde a un trabajo interinstitucional de la Secretaría de Educación Distrital y la Universidad Distrital Francisco José de Caldas, y está dirigida a la población de profesores de los colegios oficiales del Distrito Capital.

La SED considera que el trabajo en redes constituye una oportunidad sin igual para visibilizar los procesos de la labor docente a partir de la legitimación de saberes construidos, la proposición de estrategias pedagógicas, y el reconocimiento del tiempo extraescolar invertido por los docentes en actividades académicas, investigativas, de proyección a la comunidad, entre otras (SED, 2009). De ahí que esta red de maestros constituya, ante todo, un espacio de formación de formadores, reconocidos entonces como sujetos de saber y acción pedagógicos.

Hoy por hoy, esta red de maestros cuenta con 151 docentes vinculados, quienes provienen de diferentes Instituciones Educativas del Distrito Capital, correspondientes a 13 Localidades, y una mayor participación de las localidades de Bosa, Rafael Uribe, San Cristóbal y Usme.

En cuanto a la participación por género, según información recopilada durante el primer Encuentro presencial, realizado en 2010, se encuentra que hay mayor participación de mujeres $(61 \%)$ que de hombres $(39 \%)$. En relación con la formación profesional inicial que predomina en los docentes de la red, ésta corresponde a las ciencias naturales $(42 \%)$, especialmente en las áreas de biología y química.

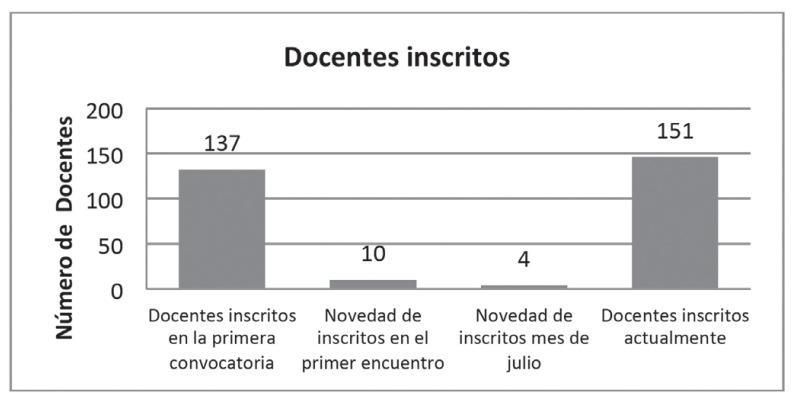

Figura 1. Relación de docentes vinculados con la Red de Maestros en Educación Ambiental del Distrito Capital. Nota. Tomado de Informe de actividades, contrato No 1471 de 2009 SED-UD, con corte a Julio de 2010

Vale la pena aclarar que también se percibe la paulatina inserción de otras áreas del conocimiento alrededor de la temática ambiental, destacándose las ciencias sociales (14\%), humanas (15\%) y áreas tecnológicas, entre otras. Este aspecto representa un indicio sobre el avance en la comprensión sistémica de lo ambiental, ya que docentes formados en diversas disciplinas encuentran un lugar común en el abordaje de temáticas ambientales y educativo ambientales. Sin embargo, se requiere más conocimiento por parte de los docentes para reconocer sus propios discursos y, de esta forma, avanzar hacia aproximaciones cognitivas desde perspectivas interdisciplinarias e integradoras.

Ahora bien, el 75\% de los integrantes de la Red de Maestros en Educación Ambiental indica poseer formación específica en el área ambiental y educativa ambiental. Al respecto, el $26 \%$ de los docentes señala que ha recibido formación ambiental en cursos, talleres, conferencias y foros; mientras que un $22 \%$ de los maestros y maestras, registra que su formación ambiental se ha dado a través de posgrados, a nivel de especializaciones $(16 \%)$ y maestrías $(6 \%)$.

Igualmente, los profesores mencionan poseer formación en el área ambiental a partir de las asignaturas cursadas durante el pregrado $(13 \%)$, a través de diplomados $(10 \%)$ y de los Programas de Formación Permanente de Docentes (PFPD), 6\%, ofrecidos por la Secretaría de Educación del Distrito. Además, algunos docentes reportan formación más de tipo empírico, lograda mediante su participación en proyectos ambientales $(10 \%)$ y durante procesos de gestión ambiental $(10 \%)$. Además, un $3 \%$ de los profesores que afirma tener este tipo de conocimiento, no específica el proceso académico que le permitió establecerlo. 


\section{Propósitos de la Red}

En cuanto a los propósitos de la Red de Maestros en Educación Ambiental del Distrito Capital, es posible encontrar como elementos fundamentales, la intencionalidad, relacionada con una cualificación y legitimación de los procesos y saberes construidos por los docentes; la incidencia de la red sobre la constitución de imaginarios, organización escolar y espacios académicos, y el impacto que pueda tener la Red de Maestros en Educación Ambiental sobre las prácticas escolares, la comunidad educativa o la sociedad en general.

Esto se propicia mediante el reconocimiento de los actores, el intercambio de conocimiento, de experiencias, de saberes y metodologías, así como a partir del uso de ejes transversales como la investigación, la interacción y la comunicación, que favorezcan la organización y armonización de procesos internos, en la perspectiva de alcanzar un amplio grado de articulación como redes y con redes afines, de carácter nacional e internacional.

\section{Las redes como estrategia en educación ambiental}

Respecto a la estrategia de formación docente a través de la Red de Maestros en Educación Ambiental, es posible afirmar que existe una sinergia que la hace pertinente, no sólo a nivel pedagógico sino educativo ambiental. Lo que significa que como red pedagógica se configura como alternativa a los programas de capacitación docente, como escenario para el fortalecimiento del maestro como sujeto de saber pedagógico y como escenario para dar alcance a políticas de Estado (Martínez, M., 2011).

Mientras que como red en el ámbito de la educación ambiental, se reconoce en múltiples escenarios como una estrategia importante para organizar y facilitar la participación ciudadana, la adhesión y el compromiso colectivo que permitan, a su vez, canalizar y conectar las necesidades, conocimientos y competencias locales, con los propios del orden regional, nacional e internacional.

En ambos escenarios, resulta clave el reconocimiento de las experiencias para retroalimentar las políticas, planes, programas y proyectos que se estén llevando a cabo. También en ambos casos, se contribuye al empoderamiento de los actores sociales involucrados, tanto en el diagnóstico de las situaciones que les afectan, como en la posibilidad de tomar las medidas necesarias para transformar esa realidad. En ese mismo sentido, se potencializa la gobernabilidad e institucionalidad, ya que las normas y las intervenciones de las entidades, provenientes de diversos sectores, pueden lograr mayor pertinencia y apropiación por parte de los agentes que las acogen, impulsan e implementan, o no.

Desde la perspectiva nacional, la formación de educadores ambientales, tiene carácter estratégico en la Política Nacional de Educación Ambiental -SINA (MEN, MMA, 2002), ya que en los Retos y Estrategias de la aludida política, Numeral 4, se señala la necesidad de dar prioridad a la formación, actualización y perfeccionamiento de los docentes y otros agentes educativo ambientales; además, se plantea como un reto "conformar una red de educadores ambientales con conexiones tanto a nivel nacional como internacional"1 ((MEN, MMA, 2002, p. 38).

En el plano internacional, se destaca el fortalecimiento y creación de redes cuando, en el Plan de Aplicación Internacional de la Década de la Educación por el Desarrollo Sostenible (2005-2014), promulgada por la Organización de las Naciones Unidas para la Educación, la Ciencia y la Cultura (UNESCO)2, se refiere como prioritario "facilitar la creación de redes, los intercambios y las interacciones entre las partes interesadas en la Educación para el Desarrollo Sostenible (EDS)".

Es en este contexto que se presenta a continuación la experiencia que, en materia de formación docente, se ha realizado en el marco de la Red de Maestros en Educación Ambiental del Distrito Capital. Esta se describe en función de los procesos pedagógicos realizados para contribuir con la formación conceptual de los profesores que se encuentran vinculados con la red.

\footnotetext{
1 En Colombia se creó la Red Colombiana de Formación Ambiental (RCFA), que hace parte de la Red Latinoamericana y del Caribe, impulsada por el Programa de Naciones Unidas para el Medio Ambiente (PNUMA). La RCFA cuenta con siete redes temáticas, entre las que se destaca la Red Temática de Educación Ambiental, a la cual se ha venido vinculando la Red de Maestros en Educación Ambiental.

2 Es necesario aclarar que este referente no se toma aquí de manera dogmática, pues aunque es cierto que la EDS es el discurso que promueve Naciones Unidas, también lo es que esta postura conlleva ambigüedades aún no resueltas, acerca del desarrollo sostenible. Por esta razón y por sus implicaciones para la región latinoamericana y del Caribe, diversos autores (González, G., 2006; Galano, 2007), prácticamente de manera contestataria, reivindican la educación ambiental como concepto por agotar y no simplemente como una figura reemplazable por acción de "instituciones colonizadas ideológica y políticamente" (González y Arias, 2009).
} 


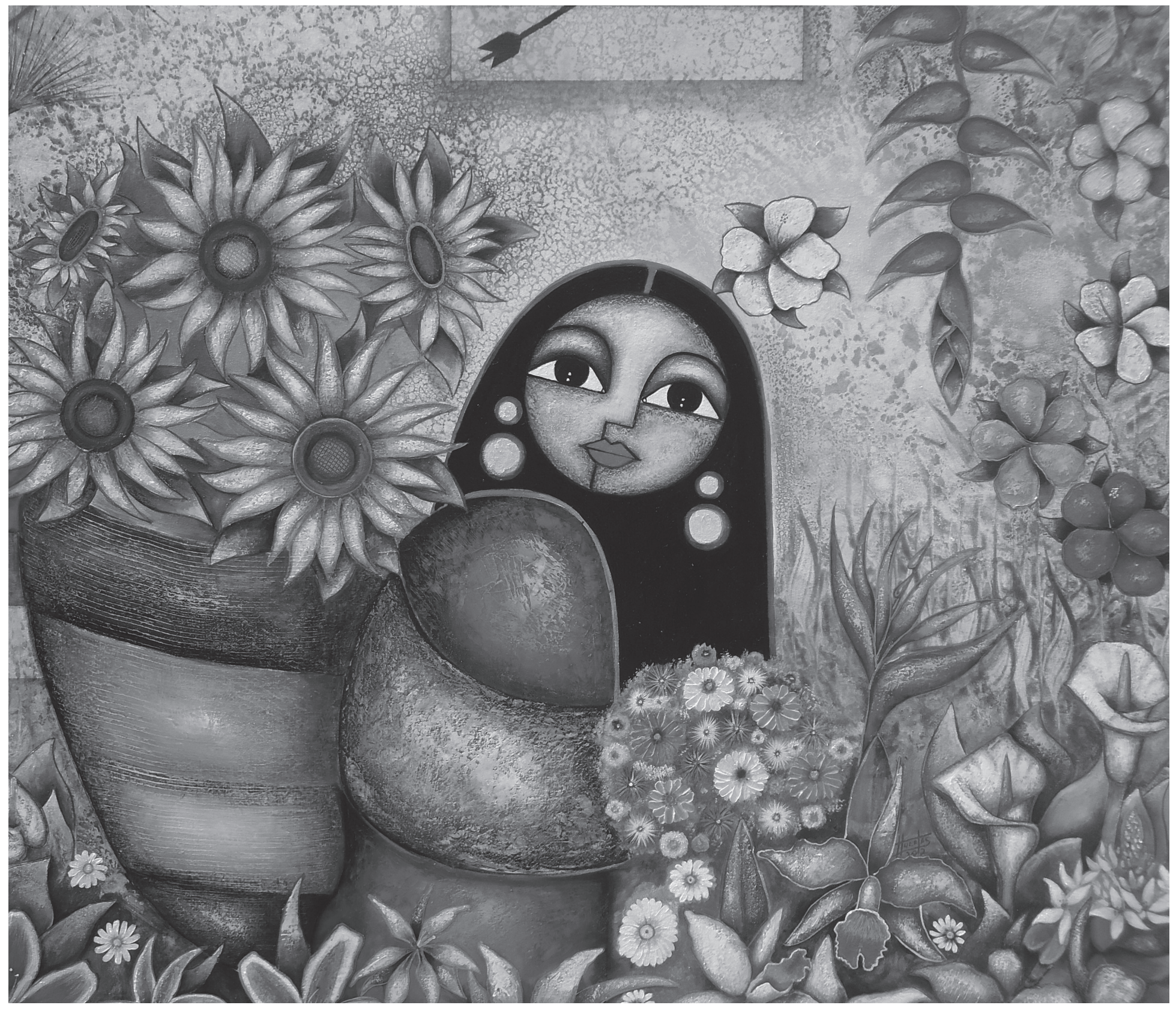

Segundo Arístides Huertas Torres ॥ Título: Jardin de ensueño ॥Técnica: Óleo sobre tela ॥ Dimensiones: $125 \mathrm{~cm}$ x $135 \mathrm{~cm}$ 


\section{Metodología}

Durante el proceso de conformación de la Red de Maestros y Maestras en Educación Ambiental del Distrito Capital, se han presentado diversas etapas metodológicas que aglutinan acciones y actividades en torno a encuentros presenciales, la formación docente, el entorno virtual y la interacción con otras redes.

Justamente, en desarrollo de esta estrategia metodológica (propia del proceso general de conformación de la red de maestros), se han implementado sincrónicamente, acciones de formación dirigidas a los profesores participantes de la red. Lo que significa que el proceso pedagógico, enfatiza las acciones realizadas en el marco de la red, con la intención de contribuir a la cualificación conceptual de sus integrantes.

En este sentido, se comenzó por la identificación de las concepciones de los docentes al ingresar a la red, mediante el establecimiento de una línea base. Posteriormente, se propiciaron actividades que les permitieran a los maestros reconocer su propio discurso y avanzar hacia la formación conceptual, por medio de la autorevisión y reflexión. Más adelante, se acudió a momentos de retroalimentación, socialización y aplicación de los avances conceptuales, mediante el intercambio e interacción logrados durante diversas actividades de la red que se aprovecharon para tal fin (Figura 2).

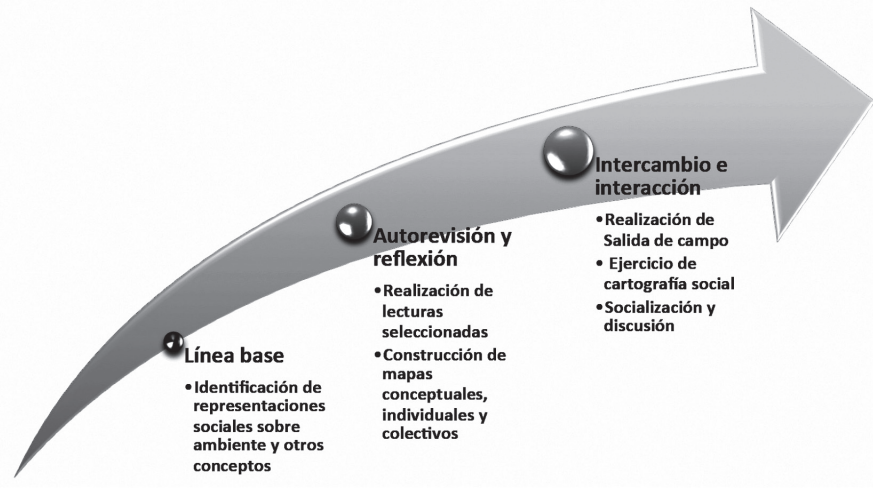

Figura 2. Estrategia metodológica de la formación docente en el marco de la conformación de la Red de Maestros en Educación Ambiental

En este orden de ideas, para recopilar la información necesaria para el establecimiento de la línea base, se aplicó una encuesta durante el primer encuentro presencial de la red de maestros, la cual diligenciaron los asistentes (30\% de profesores que integraban la red en ese momento). La información recolectada fue organizada, sistematizada y analizada utilizando elementos de investigación cualitativa, con el fin de identificar las principales tendencias de las representaciones sociales de los docentes, alrededor de conceptos como ambiente, educación ambiental y problemática ambiental.

Con base en las tendencias conceptuales identificadas en el ejercicio previo, se diseñó una estrategia metodológica para promover la autorevisión y reflexión por parte de los grupos nodales o participantes de la red, apoyada principalmente en el entorno virtual. Adicionalmente, se diseñaron y aplicaron instrumentos metodológicos para fortalecer la estrategia de formación docente en desarrollo de otras actividades de la red.

\section{Resultados}

Considerando que existe una polisemia frente al concepto de la educación ambiental a nivel mundial, traducida en diversidad de posturas epistemológicas, concepciones de ambiente, métodos pedagógicos, metodológicos e intervenciones disímiles, resulta pertinente que los integrantes de la red reconozcan sus propios discursos y tengan claridad sobre sus imaginarios, antes de entrar a interactuar con otros profesionales o colectivos. Además, se hace necesario en razón de que la postura conceptual es fundamental para abordar temáticas ambientales y educativo ambientales, pues se requieren enfoques sistémicos, integradores, holísticos, de corte inter o transdisciplinario, que reconozcan la complejidad de las interacciones entre ecosistema y cultura en contextos específicos.

\section{Línea base de concepciones sobre ambiente y educación ambiental}

Con el ánimo de contar con un nivel de referencia, se estableció la línea base de las representaciones sociales de los docentes integrantes de la red, encontrando que frente a lo ambiental, predominan las concepciones de corte biofísico y naturalista (78\%) en los maestros y maestras de la red, lo cual constituye un referente muy importante para la intervención a nivel de formación, en el sentido de llevar a los docentes a identificar variables de orden social, económico, político, ecológico, tecnológico, científico, ético, estético, entre otros, que imprimen el carácter complejo a los análisis ambientales y a los procesos educativos ambientales. 
Se evidencia, entonces, que los docentes reflexionan poco acerca del conocimiento de su objeto de estudio, en este caso, el ambiente como eje articulador de la educación ambiental, lo cual seguramente lleva a desestimar el carácter sistémico y complejo del ambiente y a abordar los procesos educativos ambientales sin considerar su naturaleza también compleja. De este modo, es muy probable que se realicen actividades que resulten en sólo buenas intenciones, pues con ellas no se aporta significativamente a atender los retos educativos, sociales o ambientales, planteados para la educación ambiental.

Aun más importante, ya que las concepciones que reflejan los profesores han sido construidas desde sus propias experiencias formativas de orden personal, profesional y social, es posible anotar que, por un lado, su práctica educativa se encuentra influenciada por tales concepciones $y$, de esta forma, reproduce prácticas insustentables ambientalmente, carentes de elementos pedagógicos, conceptuales o epistemológicos, asociados a la complejidad, visión sistémica e inter y transdisciplinaria, entre otros. Por otro lado, las concepciones de los profesores indagados expresan el discurso circulante en otros sectores de la sociedad y explican en alguna medida, los discretos avances en materia de comprensión de la dimensión ambiental del desarrollo y de la complejidad ambiental.

En la Figura 3, se indican las principales tendencias sobre la concepción de ambiente de los docentes de la Red, identificadas a partir de la organización, clasificación, análisis y categorización de las respuestas dadas por ellos. Con base en este proceso de investigación cualitativa y en otros estudios (Pérez, Porras y González, 2007; Calixto Flores, 2008; Eschenhagen, 2009), aquí se reconocen tres categorías de concepciones de ambiente: biofísica/naturalista, sistémica/globalizante y simplificadora.

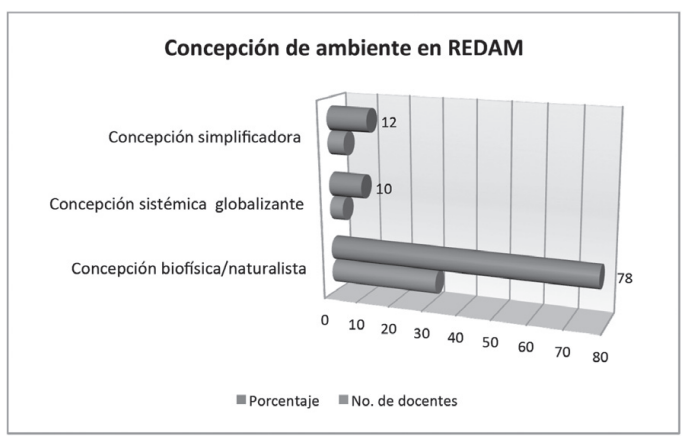

Figura 3. Concepciones sobre ambiente en docentes de la Red de Maestros en Educación Ambiental del Distrito Capital
Concepción biofísica/naturalista: en esta categoría se clasifican las respuestas que registran elementos naturales, condiciones de tipo biológico, físico o químico y sus interacciones; también las que hacen referencia a espacio físico y a las relaciones entre distintos aspectos físicos y biológicos. Se incluyen respuestas que evidencian antropocentrismo, en tanto que estos elementos interactúan en función del ser humano y su bienestar.

Concepción sistémica/globalizante: a esta categoría pertenecen las respuestas que expresan relaciones entre componentes de un sistema ambiental conformado por la sociedad, la cultura, los ecosistemas o múltiples interacciones que se establecen entre dichos conjuntos o subsistemas. Se excluyen respuestas que aluden a conjuntos de elementos biofísicos solamente.

Concepción simplificadora: en esta categoría se ubican respuestas que no dan indicio de comprensión del concepto de ambiente, pues con frecuencia se representa al mismo como "todo lo que nos rodea", sin mencionar más elementos o desarrollar la idea a la que se hace referencia con esa definición.

Respecto a las concepciones sobre educación ambiental, presentes en docentes de la Red de Maestros en Educación Ambiental del Distrito Capital (Figura 4), y con base en la clasificación propuesta por Terrón y González (2009), Sauvé (2003) y las tendencias halladas en esta investigación, se identifican las siguientes categorías: reducidas o simples, globalizadoras, antropocéntricas e integrales.

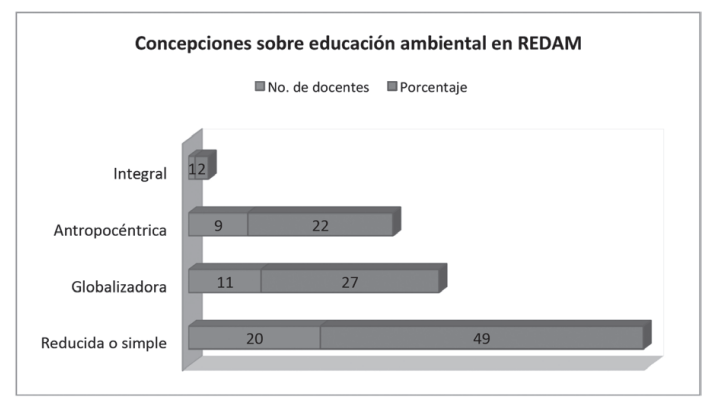

Figura 4. Concepciones sobre educación ambiental en docentes de la Red de Maestros en Educación Ambiental del Distrito Capital

Concepción reducida o simple: en esta categoría se reúnen respuestas que señalan como fines de la educación ambiental el cuidado, respeto, valoración y conocimiento de la naturaleza. Igualmente, se ubican respuestas que insinúan problemas ambientales específicos o ámbitos de acción, incluso territoriales, sin mayor alusión a factores sociales o culturales.

rollos nacionales 93 
Concepción globalizadora: para este nivel se consideran respuestas que mencionan procesos formativos para modificar relaciones sociedad naturaleza de modo muy general, también respuestas que propenden por el equilibrio y la armonía en la interacción de los seres humanos con la naturaleza, esta última asociada con la concepción biofísica de ambiente.

Concepción antropocéntrica: esta categoría agrupa las respuestas que ubican la educación ambiental de manera instrumental y en función de una concepción de ambiente para administrar, por tanto, respuestas que en un sentido utilitarista y antropocéntrico, reconocen recursos que deben administrarse de manera adecuada o "racional", en beneficio de la humanidad. Del mismo modo, respuestas que localizan la educación para la solución de problemas ambientales, para el desarrollo sostenible y para la búsqueda de equilibrios en el planeta.

Concepción integral/ crítica: esta categoría centra su atención en una visión integral y sistémica del ambiente, donde la educación emerge como espacio para comprender de manera crítica las causas y consecuencias de la crisis ambiental y para construir alternativas de vida fundamentadas en la justicia, la diversidad, el respeto y un buen vivir.

Es preciso insistir en que ante el predominio de concepciones sobre la educación ambiental (Figura 4) reducidas o simples $(49 \%)$, globalizadoras $(27 \%)$ y antropocéntricas (22\%) (A las cuales subyacen prácticas, imaginarios, modelos y sistemas insustentables ambientalmente), es relevante y necesario el proceso de formación de los docentes de la Red de Maestros en Educación Ambiental. Especialmente, porque se evidencia que se desestima la educación ambiental como proceso formativo que posibilita la problematización de situaciones para lograr que a partir de la comprensión, se tome conciencia sobre esa realidad y una posición crítica ineludible que lleve a la participación y toma de decisiones $y$, en esa medida, a la transformación de realidades.

Algo similar ocurre con la concepción de problema ambiental, evidente en lo expresado por los docentes de la Red de Maestros en Educación Ambiental (Figura 5), la cual revela una noción de ambiente desde un punto de vista conservacionista y recursista, que se expresa en virtud a que los problemas ambientales se asocian a razones de tipo biofísico y en ningún momento a facto- res económicos, políticos, sociales, científicos, éticos, históricos, epistemológicos o de otra índole cultural, que serían necesarios para explicar tales problemas y dar cuenta de la crisis civilizatoria que afronta la sociedad actual.

También es recurrente la insinuación del optimismo tecnológico, gracias al cual se podrían restablecer equilibrios o recuperar la degradación y destrucción de "recursos naturales", entendidos estos dentro de la lógica economicista que convierte bienes y servicios ambientales en bienes de mercado, por tanto, mesurables y transables bajo la oferta y la demanda impuesta por sistemas económicos, políticos y sociales que así los consideran.

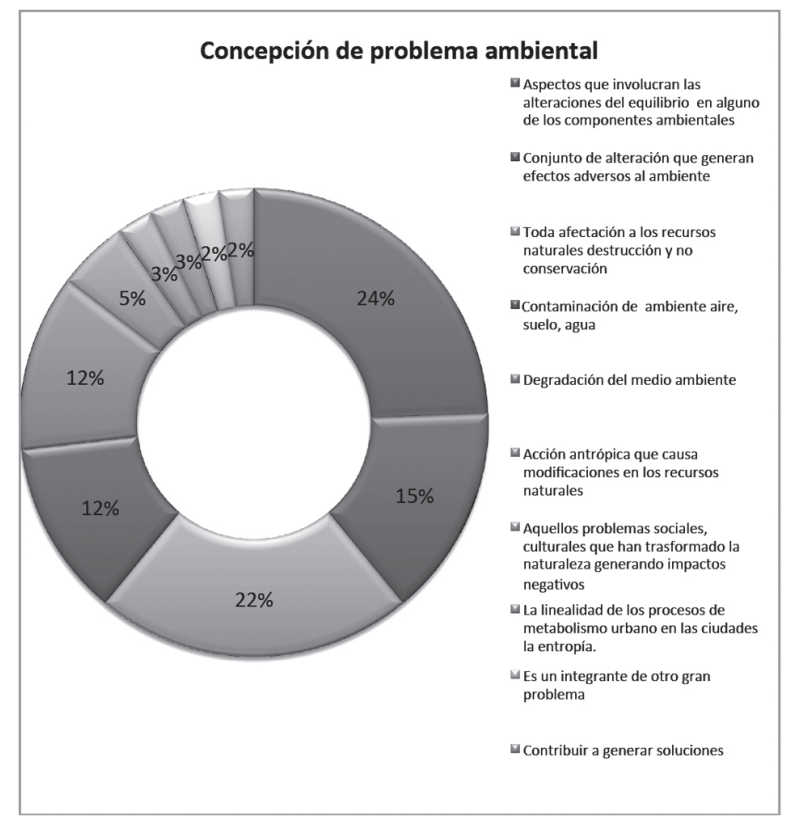

Figura 5. Concepciones sobre el problema ambiental en docentes de la Red de Maestros en Educación Ambiental del Distrito Capital. Nota. Tomada de Memorias del I Encuentro de la Red de Maestros en Educación Ambiental del Distrito Capital

En este sentido, las problemáticas ambientales y las diversas aproximaciones a su comprensión, no son mostradas aquí en el contexto de los problemas de conocimiento, a nivel de posturas epistemológicas que favorecen el antropocentrismo, la separación de los seres humanos de la naturaleza, el dominio sobre ella y la legitimación de acciones de explotación utilitarista de la misma, o desde la posición crítica frente a los desarrollos científicos y tecnológicos que refuerzan el optimismo tecnológico, el reduccionismo y positivismo de las ciencias más ortodoxas. 
Tampoco se refieren esquemas económicos o sociales que promueven mecanismos de consumo que desconocen los procesos pre y post de producción y consumo, en los cuales elementos naturales, bienes y servicios ambientales ingresan a los sistemas productivos como materia prima para ser transformados por medio de diferentes procesos, los cuales consumen más bienes y servicios, producen desechos e implican transporte, comercialización y otros procedimientos que son desestimados en la vida útil que se da a los productos y en el manejo postconsumo que se reduce a su disposición en basureros.

Finalmente, se resalta que un porcentaje representativo de participantes de la red (75\%) señaló poseer formación específica en el área ambiental y educativa ambiental, luego, se esperaría que las representaciones sociales de lo ambiental y educativo ambiental incorporaran elementos culturales (economía, política, sociedad, educación, tecnología, ciencia, ética y valores, formas de construir conocimiento y otros) de manera explícita y reflejarán su naturaleza compleja.

Por lo tanto, esta situación representa una oportunidad y una necesidad de profundizar sobre las representaciones sociales de ambiente y educación ambiental que se dinamizan a través de las estrategias de formación de formadores en la ciudad o el país, pues como se evidencia a partir de los hallazgos de este trabajo, los factores culturales son desestimados por la mayor parte de los docentes que han participado en ellos.

\section{Autorevisión y reflexión}

Teniendo en cuenta las concepciones identificadas, en los términos en que se revisan previamente, se continuó el proceso de formación de docentes en el marco de las acciones de la Red de Maestros con énfasis en educación ambiental. Para tal efecto, fue trazada una estrategia de trabajo autónomo facilitado por el entorno virtual y por el uso de los recursos de las Tecnologías de la Información y la Comunicación (TIC), donde los docentes tuvieron contacto con documentos que por su contenido y la idoneidad de sus autores (autoridades en el tema como Augusto Ángel Maya y Lucié Sauvé) representan un nivel ineluctable en la comprensión de las temáticas ambientales y educativas ambientales.

Estos documentos fueron publicados en la sección de "Lecturas básicas" del portal Web de la Red de Maestros en Educación Ambiental, disponible en http://readud. byethost6.com/, con el fin de propiciar que los maestros hicieran la lectura crítica y reflexiva de los documentos seleccionados. De manera simultánea, fue publicado un instructivo para orientar la elaboración de mapas conceptuales de las lecturas seleccionadas, el cual incluía recomendaciones sobre el uso de herramientas tecnológicas que facilitan tal labor, algunas disponibles gratuitamente en Internet, como Map Tools y Cmap Tools.

En la perspectiva de propiciar un espacio de comprensión, análisis, discusión y consensos conceptuales, en un primer momento, los profesores de la red debían construir sus mapas conceptuales de forma individual y, en un segundo momento, de manera colectiva, debían analizar y discutir los enfoques conceptuales utilizando como insumo las elaboraciones individuales.

Pese a la intencionalidad del ejercicio, el interés y avances manifestados por varios integrantes de la red, el alcance de esta actividad de formación fue limitado por factores como la disponibilidad de tiempo de los maestros (principal obstáculo referido por ellos mismos), el carácter autónomo y virtual de la actividad (que en ocasiones intimida o inhibe su participación), la limitada posibilidad de seguimiento por la propia naturaleza de la red que se desarrolla en tiempo extracurricular de los docentes y que se encuentra en un nivel primario de organización.

Sin embargo, se buscaron formas de atenuar estas condiciones limitantes por medio de la retroalimentación a través del correo electrónico, con la participación de los docentes en los Chats y foros de discusión programados en el portal Web y con la aplicación de los adelantos conceptuales en escenarios presenciales, tal como una salida de campo que se realizó como actividad de la red de maestros.

\section{Intercambio e Interacción}

Para continuar el proceso de formación de maestros y maestras de la red, durante la salida de campo al Parque Ecológico Distrital Aula Ambiental Humedal Santa María del Lago, realizada con el objetivo de generar un espacio de interacción e intercambio entre los integrantes de la red, se hizo énfasis en la discusión, análisis y reflexión frente a la concepción de ambiente y educación ambiental que cada integrante poseía y, además, se realizó un ejercicio de cartografía social. 
Para el desarrollo de la actividad, con el apoyo del facilitador designado por la Secretaría Distrital de Ambiente (SDA) para acompañar y orientar la exploración, fue realizado un recorrido por los senderos del Humedal y alrededor de su espejo de agua, donde fue resaltada la interacción de elementos históricos, sociales, económicos, institucionales, pedagógicos, legislativos, ecológicos y otros, evidentes en este ecosistema.

También fue realizado un ejercicio de cartografía social que buscaba aplicar los avances conceptuales en el área ambiental e incentivar la interacción entre los asistentes a partir de sus percepciones frente al territorio analizado; además, se practicó el ejercicio teniendo en cuenta que la base conceptual de la cartografía social reconoce, según la trilogía de Lefebvre (Soja, 1996, citado por Barrera Lobatón, S., 2009): el "espacio percibido" (o primer espacio) que corresponde al espacio material, el "espacio concebido" (o segundo espacio) referido al espacio mental y el "espacio vivido" (o tercer espacio) relacionado con las experiencias.

De suerte que un grupo de maestros y maestras, plasmaron en el mapa factores ecológicos presentes en el ecosistema estudiado, resaltando las relaciones entre factores biológicos, físicos y químicos que, vistos de manera sistémica, dan cuenta de la dimensión ecosistémica del humedal, superando la mera mención de especies de animales y plantas presentes (algunas de ellas endémicas) que resultarían en un listado y una manera simplificadora de observar este contexto.

Otros maestros y maestras identificaron el contexto histórico del humedal, señalando como éste se ve afectado por el proceso de urbanización y por las intervenciones de diversos sectores de la sociedad que determinan las presiones actuales sobre el mismo. También se destaca la interacción de lo local, lo nacional y lo global en tanto que son ámbitos que se afectan recíprocamente alterando, a su vez, el bienestar de las personas.

En cuanto a factores de tipo social, los docentes centraron su atención en las relaciones que establecen los habitantes del sector con el humedal, tanto por el significado de sus componentes (espejo de agua, senderos, rejas, otros), como por los usos establecidos para su protección. También se identificaron actores sociales e institucionales destacados en los procesos de recuperación y la constitución como aula ambiental y como parque ecológico distrital.
Desde el punto de vista económico, los maestros y maestras representaron un subsistema que interactúa con otros subsistemas y que está determinado por el carácter político de lo ambiental, manifiesto en este caso por las declaraciones de manejo y uso sostenible, de preservación de flora y fauna y de reconocimiento de valor ecológico. Igualmente, representaron servicios ambientales que presta el humedal, los cuales son susceptibles de valorarse económicamente para reconocer la importancia del humedal desde esta perspectiva.

Así mismo, desde la dimensión educativa ambiental, los maestros y maestras representaron el humedal como un escenario para la pedagogía ambiental, en el cual las distintas disciplinas y áreas del conocimiento aportan para el reconocimiento territorial. El trabajo realizado durante el levantamiento de estos mapas socialmente construidos, su socialización, discusión y análisis, permitió aproximarse a identificar el carácter sistémico (Figura 6) y complejo de las interrelaciones sociedad naturaleza que se evidencian en la conformación y reconocimiento del Humedal como aula ambiental, como zona de reserva y como parque ecológico distrital, entre otros.

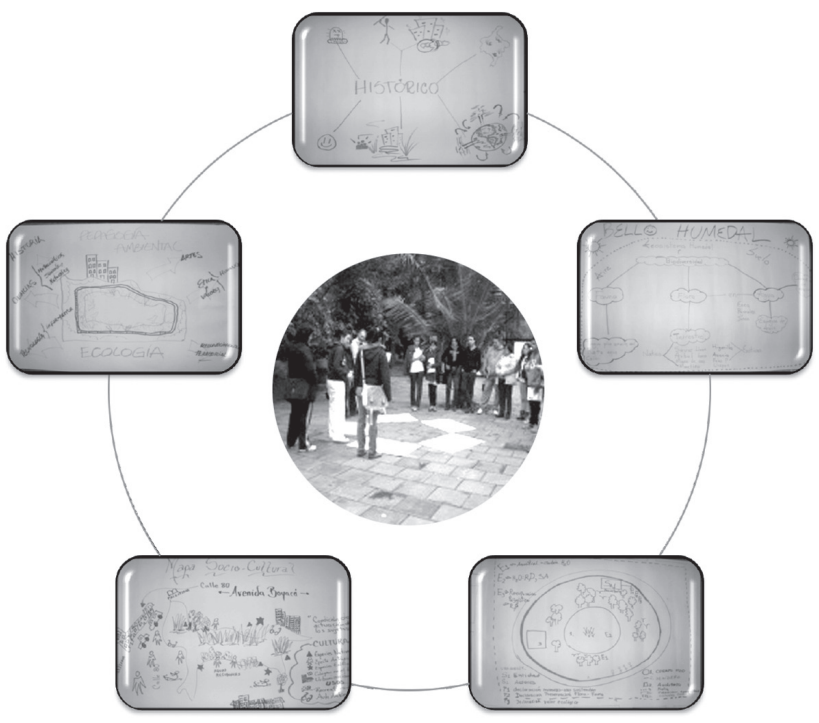

Figura 6. Aproximación a la visión sistémica del Humedal Santa María del Lago

Como saldo pedagógico de esta actividad, se evidencia, en términos conceptuales, un avance en la formación de los integrantes de la red, en cuanto a su aproximación a: comprender el ambiente desde una perspectiva 


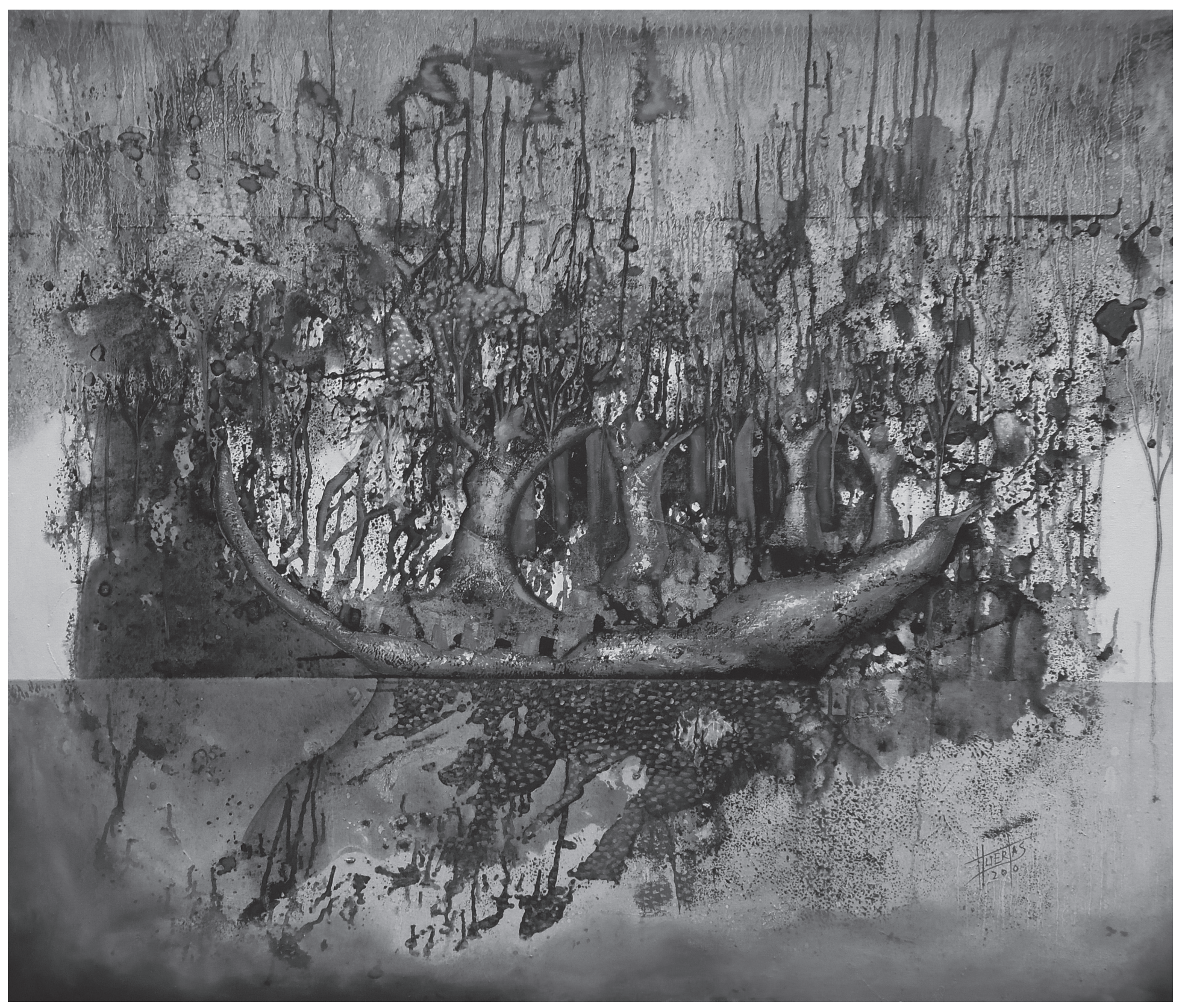

Segundo Arístides Huertas Torres ॥ Título: Paisaje Onirico ॥Técnica: Mixta sobre tela ॥ Dimensiones: $100 \mathrm{~cm}$ x $110 \mathrm{~cm}$ 
sistémica en la que múltiples interacciones definen su carácter complejo, y a visibilizar las variables de tipo cultural (económicas, políticas, sociales, educativas, científicas, tecnológicas, éticas, estéticas, entre otras) que determinan la transformación de ecosistemas.

Sin embargo, el alcance de esta actividad de la red, en sus aspiraciones de contribuir a la formación conceptual de los profesores, se vio limitada por la cobertura alcanzada en un momento determinado, puesto que los integrantes de la red no participaron masivamente en estas actividades y tuvieron un nivel de respuesta relativamente bajo ante este tipo de convocatorias presenciales.

Por otra parte, el proceso de evaluación se ha dado de manera permanente y ha permitido diseñar las estrategias metodológicas y hacer los ajustes procedimentales requeridos, según las necesidades de los maestros de la red. Aunque se hace necesario un nuevo proceso de evaluación de las representaciones sociales de los maestros, donde se contraste con la línea base establecida inicialmente, para evidenciar el impacto de la formación docente en el marco de la red. Esto también requiere que la red de maestros madure un poco más, no sólo en términos de organización como red, sino en términos de apropiación del proceso por parte de los docentes del Distrito Capital.

Vale la pena señalar que debido a que el proceso de conformación y fortalecimiento de las redes de maestros, y particularmente el de formación docente, está supeditado a las formas y dinámicas institucionales que intervienen en su configuración, existe una presión constante sobre la continuidad del proceso y sobre la posibilidad de evolucionar paulatinamente. Por lo cual, se ha insistido en avanzar hacia la generación de tejido social que garantice, en alguna medida, la autosostenibilidad y autonomía de la red, lograda a partir del conocimiento y reconocimiento entre los maestros del Distrito Capital, cuyos intereses en materia de temáticas de educación ambiental les sirvan como excusa para interactuar y fortalecerse como colectivo.

Por tanto, se espera alcanzar un mayor nivel de organización de la red, que facilite profundizar e intervenir sistemáticamente en la formación de docentes, pues es evidente que a través de su formación se contribuye en la constitución de sociedades más conscientes y responsables de su interacción con los ecosistemas que soportan la vida en el planeta Tierra.

\section{Conclusiones}

Este ejercicio permitió conocer las representaciones sociales sobre ambiente y educación ambiental que evidencian integrantes de la Red de Maestros en Educación Ambiental, a partir de las cuales se definen sus actitudes frente al ambiente, sus prácticas educativas y, en general, sus acciones conceptuales, metodológicas y pedagógicas en espacios de educación ambiental.

Los resultados señalan que sobre el concepto de ambiente, predominan las representaciones de corte biofísico y naturalista que resaltan los factores físicos, químicos y biológicos, desestimando la dimensión cultural que media en la interacción de la sociedades con la naturaleza, estos es, desatendiendo la fuerza que el mundo simbólico, la organización social y la plataforma tecnológica (según la definición de cultura de Ángel Maya) imprime a la transformación de ecosistemas.

En menor escala, se encuentran representaciones de tipo sistémico/globalizante, donde ya se reconocen múltiples interacciones y se entiende el ambiente en función de múltiples relaciones entre sus componentes. En esta medida, se reconoce un avance hacia comprender la complejidad del ambiente y las aproximaciones interdisciplinarias e integrales requeridas para su análisis. No obstante estos avances, existen algunas representaciones sociales, sobre ambiente, débiles en su argumentación, las cuales desdibujan el carácter complejo del ambiente y no permiten ubicar siquiera una noción del mismo.

Al mismo tiempo, en materia de representaciones sociales sobre educación ambiental prevalecen las reducidas o simples, las globalizadoras y antropocéntricas, muy relacionadas con la concepción biofísica y naturalista de ambiente, lo cual representa un punto de reflexión importante con los participantes de la red, ya que desde estas perspectivas es muy discreto, si no nulo, el aporte que puede tener una educación ambiental que elude sus retos a nivel social, educativo y ambiental.

En este contexto, es importante revisar las representaciones sociales que se dinamizan en virtud a los procesos de formación de formadores, implementados en la ciudad o en el país, ya que los profesores indagados en la red manifiestan haber participado en varias modalidades de estos. 
Toda vez que estas representaciones sociales fueron identificadas, se logró orientar la estrategia metodológica para avanzar en la formación docente, especialmente de tipo conceptual, prevista en desarrollo de las actividades de conformación de la Red de Maestros y Maestras en Educación Ambiental. Sin embargo, el nivel de participación y apropiación por parte de los maestros de la red, es todavía incipiente.

Si bien es cierto que los participantes con más constancia en el proceso formativo propuesto, evidencian avances conceptuales, también lo es que un sector de los integrantes de la red permanece sin mayor modificación en sus concepciones y un tanto ajeno al proceso emprendido en la red, situación que dificulta realizar una evaluación más formal sobre los adelantos en la transformación de representaciones sociales hacia esquemas más acordes con los desafíos planteados para la educación ambiental.

Es necesario progresar en el fortalecimiento de la red, ya que las redes de maestros constituyen espacios de sin igual valor e importancia para la formación docente, sin embargo, su verdadero alcance requiere tiempos de organización y maduración que conlleven a estructuras, actividades y dinámicas que propicien la modificación de los imaginarios, de las prácticas educativas y organizacionales, que sin lugar a dudas, se expresarán en la formación de ciudadanos y sociedades.

Las estrategias de formación docente en materia de educación ambiental, revisten una importancia trascendental, en tanto que la Política Nacional de Educación Ambiental reconoce en la falta de conceptualización de los agentes dinamizadores de la educación ambiental, uno de los principales problemas para mejorar significativamente en esta área, por lo cual se deben continuar explorando alternativas para contribuir en tal sentido.

\section{Referencias}

Alcaldía Mayor de Bogotá., Secretaría de Educación Distrital. (2008). Plan Sectorial de Educación 2008 - 2012: Educación de calidad para una Bogotá Positiva. Bogotá, D. C.

Ángel, A. (1998). El Retorno a la Tierra: Introducción a un método de interpretación ambiental. Serie Documentos Especiales. Bogotá: MEN, IDEA-UN, MMA. ICFES.

Barrera, S. (2009). Reflexiones sobre Sistemas de Información Geográfica Participativos (SIGP) y cartografía social. Cuadernos de Geografía, Revista Colombiana de Geografía, 18, 9-23.
Calixto, R. (2008). Representaciones sociales del medio ambiente. Perfiles Educativos. Vol. XXX. No. 120. México: Universidad Autónoma de México, 32-62.

Eschenhaghen, M. (2009). ¿Se ha logrado la integración de la educación ambiental en las universidades, especialmente en las ciencias sociales? Trabajo presentado en el $\vee$ Seminario Internacional Universidad y Ambiente. Cartagena.

Galano, C. (2007). Educación ambiental: morada de la vida. Anales de la Educación Común. Tercer siglo. Vol. 3. No. 8. Educación y Ambiente. Buenos Aires: Publicación de la Dirección General de Cultura y Educación de la Provincia de Buenos Aires, 26- 33.

González, E., y Arias, M. A. (2009). La educación ambiental institucionalizada: actos fallidos y horizontes de posibilidad. Perfiles Educativos. Vol. XXXI. No. 124. México: ISSUE-UNAM, 58-68.

González, E. (2006). Configuración y significado: educación para el desarrollo sustentable. Trayectorias, VIII, (20-21), 100 - 109.

Martínez, C. (2011). Trayectoria y potencia de las redes pedagógicas de maestros y maestras en Colombia. Palabra Maestra: publicación del Premio Compartir al Maestro. Obtenido desde, http:/palabramaestra.premiocompartiralmaestro.org/notitotal.php

Pérez, R., Porras, Y., y González, A. (2007). Identificación de las representaciones de ambiente y educación ambiental que circulan en la escuela. Revista Tecne, Epsiteme y Didaxis-TED, 21, 24-44.

República de Colombia. Ministerio de Educación Nacional y Ministerio del Medio Ambiente. (2002) Política Nacional de Educación Ambiental SINA. Bogotá.

Sauvé, L. (2003). Perspectivas curriculares para la formación de formadores en educación ambiental. Memorias Primer Foro Nacional sobre la Incorporación de la perspectiva Ambiental en la formación técnica y profesional. México: Universidad Autónoma de San Luis de Potosí.

Terrón, E., y González, E. (2009). Representación y medio ambiente en la educación básica en México. Trayectorias, 11 (28), $58-81$.

UNESCO. (2006). Decenio de la Educación para el Desarrollo Sostenible: Plan de Aplicación Internacional. París: Sección de la educación para el desarrollo sostenible (ED/UNP/ESD).

Universidad Distrital Francisco José de Caldas. (2010). Informe de actividades Contrato No. 1471 de 2009 SED-UD. Bogotá: Proyecto Red de Maestros en Educación Ambiental SED-UD, 1-55.

Universidad Distrital Francisco José de Caldas. (2010). Memorias del I encuentro de la Red de Maestros en Educación Ambiental del Distrito Capital. Bogotá: Proyecto Red de Maestros en Educación Ambiental SED-UD, 2-98.

Zamudio, C. (2009). Red de Educadores Ambientales Distritales. Memorias VI Congreso Iberoamericano de Educación Ambiental. Argentina. 


\section{Dialogo del conocimiento}

El artículo de Carmen Zamudio Red de Maestros en Educación Ambiental: un espacio de formación de formadores en Bogotá, Colombia trabaja tres aspectos sumamente relevantes: la formación de formadores, la articulación de docentes a través del enfoque de redes y las representaciones que estos tienen sobre la educación ambiental.

Sobre el último elemento, desde los años setenta, pero principalmente desde la década de los años noventa, la educación ambiental cobró relevancia y se constituyó en un tema de obligada referencia en las instituciones educativas, con la intención de reconocer las problemáticas ambientales que ya estaban evidenciándose como caóticas. Sin embargo, después de 20 años de discusiones al respecto, la situación ambiental se ha deteriorado de manera dramática y la educación no parece haber dado respuestas contundentes para enfrentar la crisis.

La realidad ambiental actual, caracterizada por el cambio climático, la excesiva producción de desechos, la apropiación de la naturaleza por parte de multinacionales como base para su explotación en el mercado; la agroindustria y los concomitantes fertilizantes, fungicidas, herbicidas, etc. que son utilizados para la producción de alimentos a gran escala, bajo el modelo del monocultivo; el deterioro de la socio y la biodiversidad; la megaminería, responsable de la contaminación de fuentes de agua, de la pérdida de zonas de reserva natural protegidas - fauna y flora devastadas-; la perdida de los ecosistemas, así como el aumento de la inequidad y la pobreza, el individualismo, la injusticia social, entre muchos otros fenómenos, plantean una alerta roja en el tema ambiental, pero sobre todo lo desbordan, pues la amenaza es para la vida humana y no solo para la naturaleza o aquello que se ha concebido como externo al hombre, llamado ambiente.

Desde lo señalado, es paradójico o sorprendente que, como lo muestra el artículo de Carmen Zamudio a partir de la línea de base construida en el proceso de trabajo en la Red, los maestros comprometidos con la temática no reconocen la complejidad de la situación, en principio la mayoría de ellos reduce lo ambiental a asuntos de orden biofísico o químico y sus interacciones, al igual que asumen, en algunos casos, que la tecnología será suficiente para superar los desmanes de una sociedad que actúa bajo un modelo de desarrollo insostenible; de todo lo cual se infiere que desconocen u omiten elementos como los mencionados en el párrafo anterior, obvian procesos de orden político, social, económico, y en últimas que no conciben lo ambiental desde una perspectiva sistémica, inter y transdisciplinar, como se expone en el artículo.

Así mismo, es llamativo que los procesos formativos posteriores que se desarrollaron en la Red para aportar en la cualificación del saber de los docentes, aunque involucraron lo virtual, lo experiencial, propiciaron la construcción de una cartografía social a partir de la interacción entre los mismos docentes, y el reconocimiento de sus percepciones, que si bien constituyeron elementos de reflexión y construcción de nuevos enfoques, solo impactaron un número muy bajo de docentes, pues por diversas razones la mayoría de miembros, no participó en las experiencias pedagógicas desplegadas para complejizar los conceptos y enriquecer las perspectivas iniciales.

De acuerdo a lo anterior, surgen distintas inquietudes de la lectura del texto de la profesora Zamudio. En primer lugar, genera preocupación el hecho de no comprender cómo quienes están más involucrados en los temas ambientales en los espacios educativos, obvien la relevancia del mismo modelo de desarrollo y de los asuntos político-económicos en el deterioro de los entornos vitales, pues socialmente han asumido el compromiso de formar e informar, de posibilitar transformaciones, de coadyuvar a enfrentar dicha crisis. Pero además impacta que desconozcan las dimensiones que hoy tiene la situación ambiental en nuestro país, pues esta compromete la tenencia de la tierra, el acceso al agua -líquido vital- y finalmente implica la sostenibilidad del mismo futuro.

Por otra parte, también inquieta el tratamiento de la educación ambiental en los espacios educativos, pues como eje trasversal en la formación de niños, adolescentes y jóvenes, parece no tener mayor relevancia, ni espacios de discusión y real fundamentación, ya que de acuerdo a los hallazgos acerca de las concepciones de la educación ambiental, el enfoque menos considerado por los maestros es el "integral-crítico", lo cual implica que la apuesta por construir desde la educación una mirada reflexiva, comprometida con la resolución de problemáticas que involucran la acción humana, más allá de las acciones planas del reciclaje, sin contexto, ni debate, no está siendo considerada. De esta manera, preocupan los procesos que se están generando en los espacios educativos, las reflexiones y las acciones que se están contribuyendo a movilizar.

Finalmente, es cuestionador, desde lo que se percibe en el artículo, que pese a la fuerza de la Red como ámbito articulador de intereses, núcleo dinamizador de propuestas de formación de formadores, la transformación de las concepciones de los docentes, el interés por parte de estos en comprender -desde una posición crítica- las problemáticas ambientales y el compromiso con su resolución, se vislumbren demasiado inciertas; lo cual a su vez pone en riesgo la posibilidad de que en la escuela se abogue por la formación de sujetos críticos, activos, cocreadores de alternativas a la crisis que más que ambiental, es política - parafraseando a José Mujica-, la cual amenaza nuestra “civilización”.

Ingrid Delgadillo

100 nodos y nudos / volumen 4 N.ㅇ 32 / enero-junio 2012 